\title{
Parathyroid crisis in a 20 year old - an unusual cause of hypercalcaemic crisis
}

\author{
P Wong, C Carmeci, R B Jeffrey, R J Weigel
}

Abstract

Since the advent of automated serum analysis, patients with primary hyperparathyroidism (PHPT) are often asymptomatic at presentation or have mild symptoms attributable to the disease. Parathyroid crisis is a rare and potentially fatal complication of PHPT in which patients develop severe hypercalcaemia with signs and symptoms of multiple organ dysfunction. A case of parathyroid crisis in a 20 year old man who presented with brown
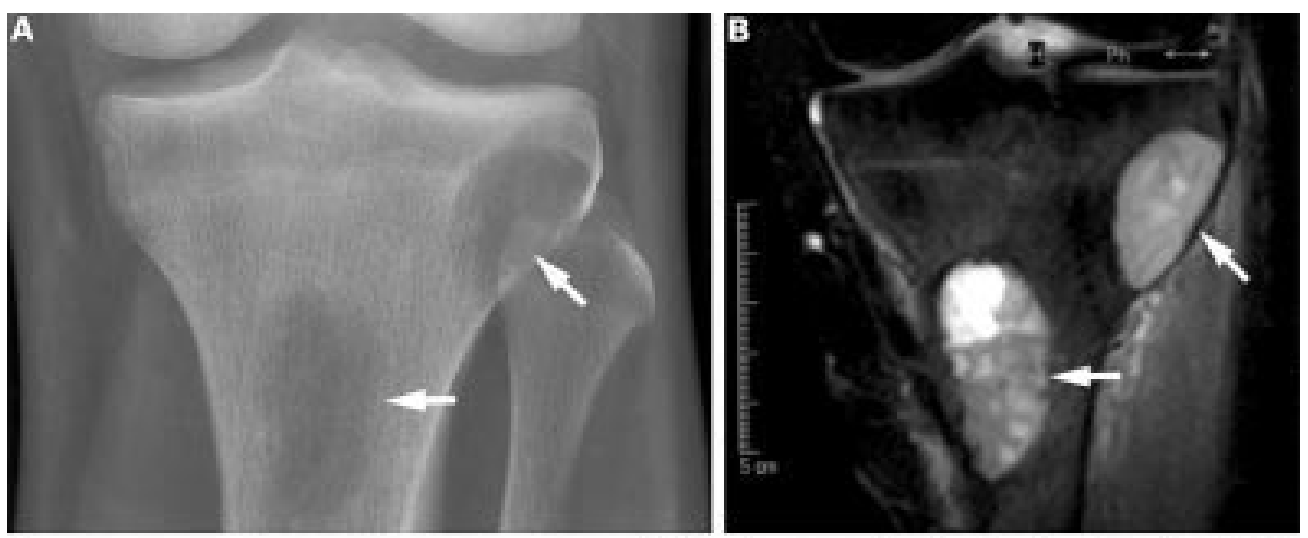

C

Stanford University School of Medicine, Stanford, California: Department of

Surgery

P Wong

C Carmeci

R J Weigel

Department of

Radiology

R B Jeffrey

Correspondence to: Dr Ronald J Weigel, MSLS, Room P214, 1201 Welch

Road, Stanford University School of Medicine,

Stanford, CA 94305-5494, USA

ronald.weigel@stanford.edu

Submitted 15 August 2000 Accepted 28 November 2000 tumours and renal stones is described.

(Postgrad Med f 2001;77:468-470)

Keywords: hypercalcaemia; hyperparathyroidism; parathyroid crisis; hypercalcaemic crisis

\section{Case report}

A 20 year old college student presented with left knee pain after a minor sport's injury. Plain radiographs of the knee demonstrated multiple lytic bone lesions in the distal femur and proximal tibia, which were confirmed by magnetic
Figure 1 Preoperative studies. (A) Plain radiograph of left knee demonstrating lytic bone lesions in the lateral aspect of the tibial epiphysis and metaphysis. (B) Magnetic resonance image demonstrating two lesions in the proximal tibia each approximately $4 \times 2 \mathrm{~cm}$ which were hyperintense on $T 2$ weighted images. A third lesion in the femur is not shown. $(C)$ Bone scan demonstrating generalised increased uptake with focal moderate activity around the left knee. (D) High resolution ultrasound demonstrated parathyroid adenoma (arrows) inferior to left lobe of the thyroid. Lower image using Doppler demonstrated flow in vessels (arrow heads) feeding parathyroid adenoma. 


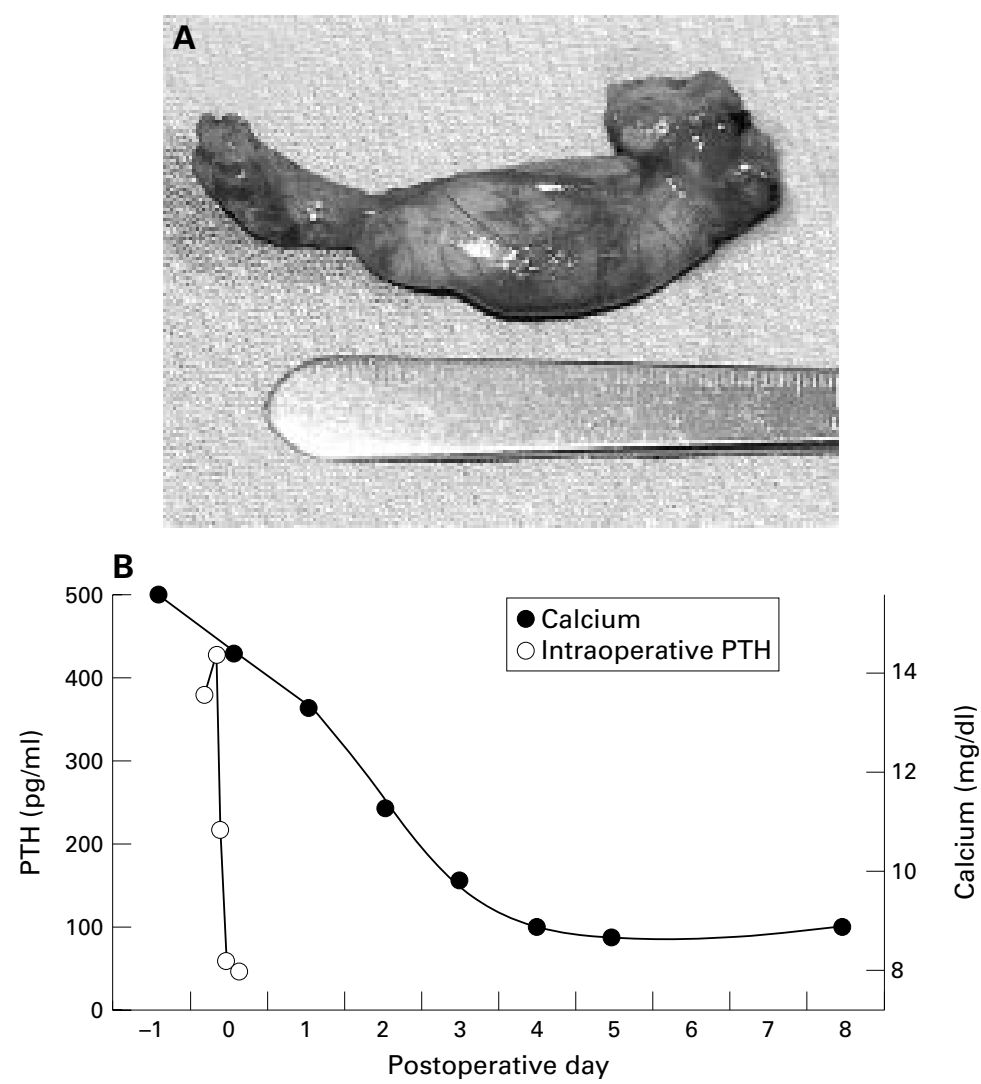

Figure 2 Intraoperative findings. (A) Large right inferior parathyroid adenoma (7.92 g) resected. (B) Intraoperative PTH values ( $\mathrm{pg} / \mathrm{ml})$ were as follows: time of neck incision, 378 ; time of pedicle ligation of large right inferior gland, 426; 10 minutes after pedicle ligation, 216; 10 minutes after resection of two left parathyroid glands, 58.5; 10 minutes after neck dissection complete, 46.3 (to convert PTH from $\mathrm{pg} / \mathrm{ml}$ to pmol/l multiply by 0.1053 ). Serum calcium normalised after three days and stabilised in low-normal range (to convert calcium from $\mathrm{mg} / \mathrm{dl}$ to $\mathrm{mmol} / \mathrm{l}$ multiply by 0.250 ).

resonance imaging (figs $1 \mathrm{~A}, 1 \mathrm{~B}$ ). The lesions were hyperintense on $\mathrm{T} 2$ weighted images and isointense with marrow on $\mathrm{T} 1$ weighted images. These findings were consistent with brown tumours or a malignancy.

The patient was referred to an orthopaedic oncologist for evaluation of possible bone malignancy. Blood chemistry showed a markedly raised serum calcium of $4.0 \mathrm{mmol} / 1$ (normal 2.2-2.6) (16 mg/dl; normal 8.7-10.2), ionised calcium $2.32 \mathrm{mmol} / 1$ (1.13-1.32), phosphorus $0.7 \mathrm{mmol} / 1(0.8-1.6)(2.2 \mathrm{mg} / \mathrm{dl}$; 2.5-4.9), and alkaline phosphatase $413 \mathrm{U} / 1$ (50-136). Other findings were anaemia with a packed cell volume of $0.365(0.42-0.52)$ and impaired renal function with a creatinine of $141.4 \mu \mathrm{mol} / 1$ (70.7-123.8) (1.6 mg/dl; 0.8 1.4). The patient was admitted as an emergency for hypercalcaemic crisis. $\mathrm{He}$ was promptly treated with aggressive intravenous hydration with normal saline, frusemide (furosemide) diuresis, and etidronate. A bone scan showed diffuse increase in metabolic activity, with intense foci at the left knee (fig 1C). His intact parathyroid hormone (PTH) concentration was markedly raised at $116.9 \mathrm{pmol} / 1$ (normal 1.3-7.6).

The patient was transferred to our hospital for further evaluation and management. Careful questioning revealed a two year history of increasing fatigue, headaches, light sensitivity, polydypsia, and polyuria. He had a one month history of nausea with vomiting usually upon awakening. The patient's mother reported that the patient seemed irritable and depressed. Blood chemistry demonstrated a raised total calcium of $3.8 \mathrm{mmol} / 1(15.3 \mathrm{mg} / \mathrm{dl})$ and creatinine of $141.4 \mu \mathrm{mol} / 1(1.6 \mathrm{mg} / \mathrm{dl})$. Ultrasound of the neck identified a hypoechoic, hypervascular, ovoid mass inferior to the right lobe of the thyroid consistent with a parathyroid adenoma (fig 1D).

The patient underwent a bilateral neck exploration that identified four gland parathyroid hyperplasia. The largest gland was the right inferior parathyroid, which weighed 7.92 $\mathrm{g}$ and extended into the mediastinum (fig 2A). Several large feeding arteries and veins were identified. Three parathyroid glands were resected and the right superior parathyroid gland was generously biopsied leaving approximately $60 \mathrm{mg}$ of parathyroid tissue. Intraoperative PTH concentrations were evaluated during neck dissection (fig 2B). PTH concentrations normalised 10 minutes after neck dissection was completed and serum calcium normalised within three days (fig 2B).

The patient's creatinine peaked at 194.5 $\mu \mathrm{mol} / 1(2.2 \mathrm{mg} / \mathrm{dl})$ and evaluation with a renal ultrasound and computed tomography revealed multiple stones, diffuse nephrocalcinosis, and mild hydronephrosis of the right kidney. Haematological evaluation revealed a normocytic, normochromic anaemia. The patient was discharged on $500 \mathrm{mg}$ of calcium daily. One month postoperatively, his serum calcium was $2.1 \mathrm{mmol} / 1(8.4 \mathrm{mg} / \mathrm{dl})$ and creatinine was normal $(106.0 \mu \mathrm{mol} / \mathrm{l} ; 1.2 \mathrm{mg} / \mathrm{dl})$. He reported resolution of his fevers, fatigue, vomiting, and arthralgias.

\section{Discussion}

Hypercalcaemic crisis is characterised by severe hypercalcaemia, usually with a calcium $>3.5 \mathrm{mmol} / \mathrm{l}(>14 \mathrm{mg} / \mathrm{dl})$, with associated signs and symptoms involving multiple organ systems. ${ }^{1-3}$ Patients often display metabolic encephalopathy, renal insufficiency, gastrointestinal symptoms, and cardiac dysrhythmia. ${ }^{2}$ Malignancy is the most common cause of hypercalcaemic crisis, which results from secretion of PTH related polypeptide or from bone resorption related to osseous metastases. ${ }^{2}$ Cancers most commonly associated with hypercalcaemia include squamous cell carcinoma of the lung, breast cancer, renal cell carcinoma, bladder cancer, and multiple myeloma.

Hypercalcaemia occurring with a raised intact PTH is pathognomonic for primary hyperparathyroidism (PHPT). ${ }^{1}$ Severe hypercalcaemia occurs in $1 \%-2 \%$ of patients with PHPT and has been referred to as acute hyperparathyroidism, parathyroid crisis, parathyroid storm, and parathyrotoxicosis. ${ }^{1}$ Other complications which are common in patients with parathyroid crisis include kidney stones, renal insufficiency, brown tumours, proximal muscle weakness, neuropsychiatric syndrome with decreased ability to concentrate and depression, and a normochromic, normocytic anaemia. ${ }^{13-7}$ Hypercalcaemic crisis has been reported to be as high as $50 \%$ in children age 
18 or younger. ${ }^{8}$ It is unclear whether the higher rate of crisis in the paediatric population is due to delayed diagnosis or a more aggressive form of the disease. ${ }^{9}$ The occurrence of hyperparathyroidism at an early age, as in this patient, should also raise the suspicion of multiple endocrine neoplasia type 1 .

The initial treatment of hypercalcaemic crisis is the same regardless of the aetiology. Therapy is aimed at increasing renal excretion of calcium and decreasing bone resorption. ${ }^{1-3}$ Patients with hypercalcaemic crisis are volume depleted and initial therapy should be intravenous hydration with isotonic saline. The conventional use of loop diuretics to promote calciuresis is currently under scrutiny as this may exacerbate extracellular volume depletion through concomitant sodium diuresis. ${ }^{2}{ }^{3}$ In patients with renal failure, dialysis with a low or zero calcium dialysate should be initiated. ${ }^{10}$ Bisphosphonates, which directly inhibit osteoclast activity, are the most effective of the pharmacological agents that decrease bone resorption. Pamidronate has gained acceptance as the drug of choice since it is the most potent bisphosphonate and has the fewest side effects. ${ }^{2}$

After initial medical stabilisation, surgical exploration should be performed on patients with parathyroid crisis. Recent series have reported excellent results from urgent parathyroidectomy, with perioperative mortality of less than 5\%. ${ }^{4-7}$ As for all patients with PHPT, approximately $85 \%-90 \%$ of patients with parathyroid crisis have a single adenoma. ${ }^{4-7}$ As exemplified in our patient, serum PTH levels decline rapidly after parathyroidectomy, while serum calcium normalises over the course of several days. The correction of serum calcium and PTH is accompanied by resolution of bone pain, renal insufficiency, cardiac dysrhythmias, and mental status changes. Complete resolution of the metabolic derangements including resolution of bone, renal, and haematological abnormalities may take six to 12 months. ${ }^{3}{ }^{4}$

\section{Conclusion}

Hyperparathyroidism must be considered in the differential diagnosis for severe hypercalcaemia in all age groups. Patients can present

\section{Learning points}

- Primary hyperparathyroidism (PHPT) must be considered in the differential diagnosis for hypercalcaemic crisis.

- Hypercalcaemia in the setting of a raised intact parathyroid hormone is pathognomonic for PHPT.

- Although PHPT is rare in young adults and children, they often present with hypercalcaemic crisis.

- Parathyroid crisis requires immediate medical management for hypercalcaemia followed by urgent surgery.

- Normalisation of serum calcium concentrations leads to gradual reversal of metabolic abnormalities associated with hypercalcaemia.

with a wide range of symptoms related to metabolic derangements of multiple organ systems. Initial medical management is aimed at lowering serum calcium by increasing renal excretion of calcium and reducing bone resorption. Patients should be treated by urgent parathyroidectomy. Surgical treatment results in prompt correction of serum calcium and PTH with gradual resolution of the metabolic consequences of severe hypercalcaemia.

1 Kebebew E, Clark OH. Parathyroid adenoma, hyperplasia, and carcinoma. Surg Oncol Clin North Am 1998;7:721-48. Edelson GW, Kleerekoper M. Hypercalcemic crisis. Med Clin North Am 1995;79:79-92.

3 Wilson JD. Williams textbook of endocrinology. 9th Ed. Philadelphia: WB Saunders, 1998.

4 Wang CA, Guyton SW. Hyperparathyroid crisis: clinical and Wang CA, Guyton SW. Hyperparathyroid crisis: clinical and
pathologic studies of 14 patients. Ann Surg 1979;190:782patholc

5 Fitzpatrick LA, Bilezikian JP. Acute primary hyperparathyroidism. Am f Med 1987;82:275-82.

6 Maselly MJ, Lawrence AM, Brooks M, et al. Hyperparathyroid crisis: successful treatment of ten comatose patients. Surgery 1981;90:741-6.

7 Schweitzer VG, Thompson NW, Harness JK, et al. Management of severe hypercalcemia caused by primary hyperparathyroidism. Arch Surg 1978;113:373-81.

8 Cronin CS, Reeve TS, Robinson B, et al. Primary hyperparathyroidism in childhood and adolescence. 7 Paediatr Child Health 1996;32:397-9.

9 Harman CR, vanHeerden JA, Farley DR, et al. Sporadic primary hyperparathyroidism in young patients: a separate disease entity? Arch Surg 1999;134:651-6.

10 Kaiser W, Biesenback G, Kramar R, et al. Calcium free hemodialysis: an effective therapy in hypercalcemic crisisreport of 4 cases. Intensive Care Medicine 1989;15:471-4. 